\title{
Conceptual model study using origami for membrane space structures - a perspective of origami-based engineering
}

\author{
M. C. NATORI*, Hiraku SAKAMOTO**, Nobuhisa KATSUMATA***, \\ Hiroshi YAMAKAWA**** and Naoko KISHIMOTO***** \\ *Institute of Space and Aeronautical Science (Professor Emeritus), \\ 3-1-1 Yoshinodai, Chuo-ku, Sagamihara-shi, Kanagawa 252-5210, Japan \\ E-mail: mcnatori@ac.jaxa.jp \\ **Department of Mechanical and Aerospace Engineering, Tokyo Institute of Technology \\ 2-12-1 Ookayama, Meguro-ku, Tokyo 152-8552, Japan \\ ***Department of Mechanical, Aerospace, and Materials Engineering, Muroran Institute of Technology \\ 27-1 Mizumoto-cho, Muroran, Hokkaido, 050-8585, Japan

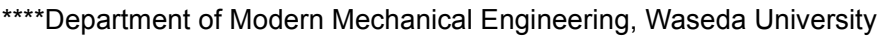 \\ 3-4-1 Okubo, Shinjuku-ku, Tokyo 169-8555, Japan \\ *****Department of Mechanical Engineering, Setsunan University \\ 17-8 Ikedanaka-machi, Neyagawa City, Osaka, 572-8508, Japan
}

Received 1 September 2014

\begin{abstract}
This paper discusses what has been found and what will be found using conceptual "origami" models to develop deployable space structures. The study covers the following: (i) one-dimensional structural elements, which are axially buckled inflatable tubes; (ii) two-dimensional elements, which are deployable membranes, such as solar arrays and solar sails; and (iii) deployable elements in nature. The study clarifies what design considerations are necessary to adapt the basic concepts to actual space structural hardware, and several limitations of origami models are discussed. Regarding the last subject, this study envisions future space structures using conceptual origami models that imitate three-dimensional deployable structures in nature, such as flowers and insect wings.
\end{abstract}

Key words : Aerospace engineering, Structural mechanics, Satellite design, Deployable structures, Folding patterns

\section{Introduction}

Because the number of actual space structures already realized is extremely small compared with normal massproduced structures on the ground, and because the launch opportunities of space structures being constructed is still limited at present, the conceptual design phases for space structures are particularly important. Due to the weight and size restrictions of transportation vehicles from ground to space, space structures must be lightweight, and in many cases, the use of a deployable function is required. To design efficient space structures, various structural hardware options must be carefully investigated from the conceptual design phase, and sometimes, the use of conceptual origami models is effective because origami models can often be easily made and modified. Also, in particular, to satisfy recent requirements of constructing structures with large apertures in space, such as solar cell arrays, solar sails, and flat phased antennas, deployable membrane structures are extremely important (Jenkins, 2006), (Natori, 2007a). Origami models are often used to study membrane space structures; however, systematic interpretations of how origami models can contribute to space structure design have been rarely discussed.

To this end, the objectives of the present paper is to clarify the findings that have been made using conceptual origami models in past space structure developments and to predict what contributions can be made for future designs of space structures. To achieve this purpose, this paper presents various examples of conceptual origami models used to design deployable space structures, mostly exemplified by the present authors. In addition, necessary considerations for applying origami models to actual mechanical hardware are discussed. 
The following items in regards to origami models are discussed in this paper with examples.

1. Method to maintain flow path for inflation gas

2. Properties of elastic deformation during deployment and stowage

3. Possibility of undesired folding/stowage patterns

4. Estimation of shape/size of stowed configuration

5. Effect of thickness during stowage

6. Generation of innovative folding patterns using a "dual" relation

7. Conception of future space structures by learning from nature

To the best of the authors' knowledge, no other researcher has attempted to categorize the contribution of origami models to the design process of space structures.

The present paper is organized as follows, in which the discussion evolves from one-dimensional deployment of a structure to three-dimensional deployment. First, one-dimensional inflatable tube structures are discussed, and their possible stowed configurations are shown by using axially buckled cylindrical shell models. It is noted that in actual structures, elastic deformation of tube membrane realizes their deployment. Second, for planar two-dimensional deployable membrane structures, two typical folding patterns, spiral folding pattern and circumferential folding, are introduced. Their mutual relations through geometrical tessellation are explained, and many model versions, including those driven from their dual properties, are shown. The models of deployable modular structures for future large space structure systems are also presented. Finally, examples of deployable concepts in nature and their interpretation for structural mechanics, which includes the concept of growth, are introduced to obtain a variety of innovative ideas for more advanced designs of space structure systems.

\section{Origami models of axially buckled cylindrical shells}

\subsection{Deployment of inflatable tubes and modified zigzag folding pattern}

Figure 1 shows various deployment aspects of inflatable booms. An attempt to demonstrate inflatable structure technology in space was conducted in 1996 (IAE: Inflatable Antenna Experiment (Freeland, et al., 1997)) by JPL/L'Garde. The deployment behavior of its inflatable tubes is shown in Fig. 1(a). Note that deployment control of inflatable tubes folded in a zigzag pattern is extremely important because inflation gas is obstructed at the folding line; a modified zigzag folding pattern shown in Fig. 1(b) has been developed on the basis of origami, as discussed in (Katsumata, et al., 2012), in which a common cavity between the upstream and downstream sides is created at the folding line. In Figs. 1(c)-(e), deployment behaviors of inflatable booms in three different folding patterns are shown, which are made by sealing two thick aluminum-evaporated laminated films (total thickness: $76 \mu \mathrm{m}$, length: $900 \mathrm{~mm}$ ) through heating. The boom folded in the zigzag pattern also moves in the out-of-plane direction violently, which is not adequately shown in the pictures taken from upper position. The rolled-up boom deploys smoothly at first, though more slowly later, requiring a longer time to reach full deployment. In the modified zigzag pattern, some elastic deformation caused by gas

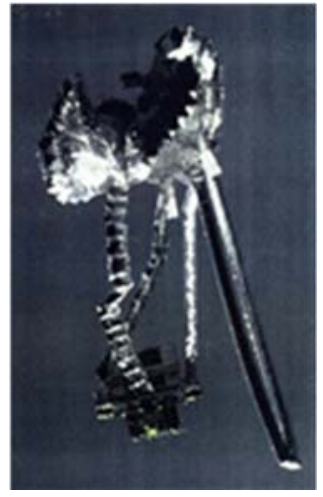

(a) Actual example in space (IAE, (Freeland, et al., 1997))

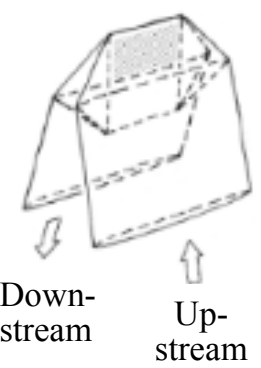

(b) Conceptual origami image of modified zigzag folding

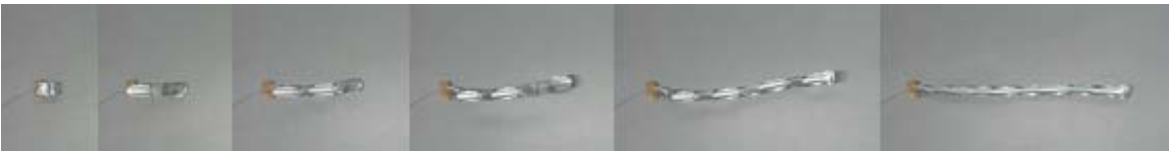

$5 \mathrm{sec}$

$0 \quad 1 \quad 2$

3

4

(c) Deployment behavior of a boom folded in a modified zigzag pattern

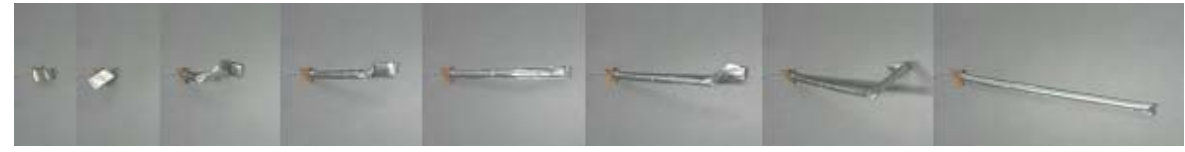
$0 \quad 1$
2
3
4
5
6
$7 \mathrm{sec}$

(d) Deployment behavior of a boom folded in a zigzag pattern

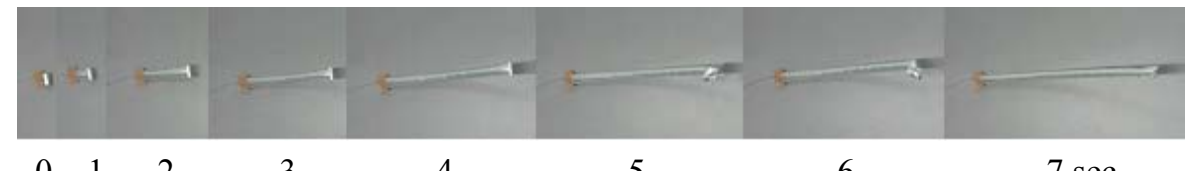

(e) Deployment behavior of a rolled-up boom

Fig. 1 Deployment of inflatable booms 


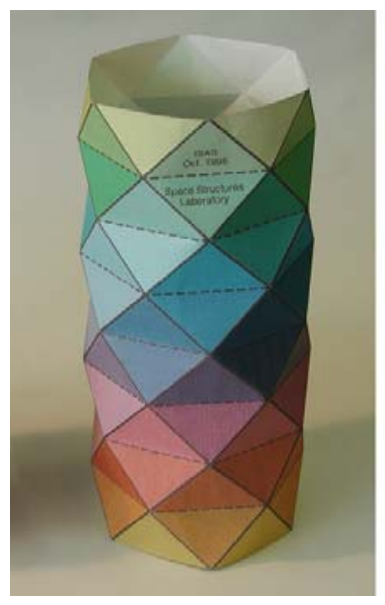

(a) Right triangle

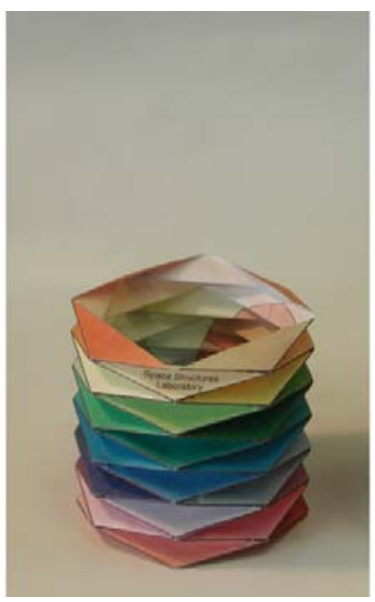

(b) Obtuse triangle, 1

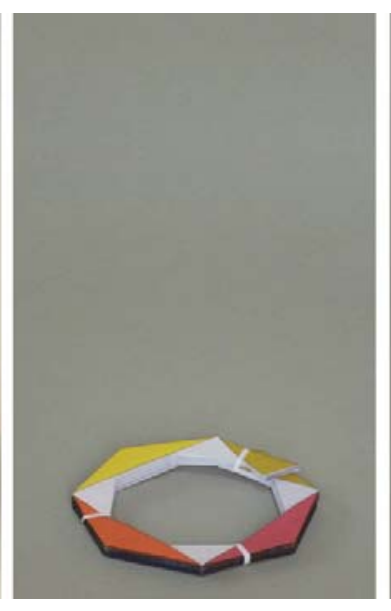

(c) Obtuse triangle, 2

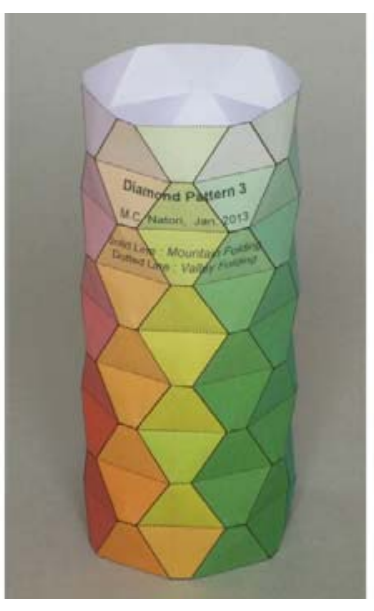

(d) Trapezoid

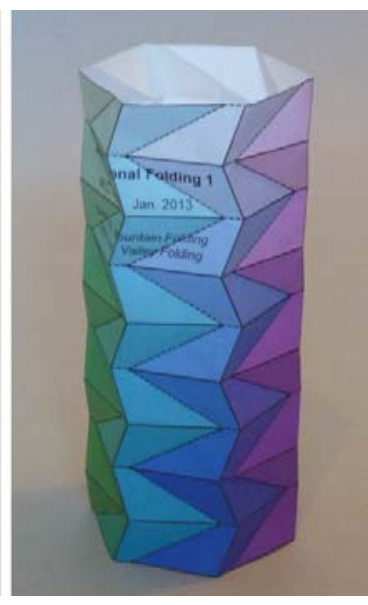

(e) Slanted trapezoid

Fig. 2 Origami models of buckled cylindrical shells using different basic polygonal shapes pressure makes the flow path simpler.

\subsection{Origami models of buckled cylindrical shells for stowed inflatable tubes}

To ensure a complete flow path, stowed configurations inspired by various patterns of buckled cylindrical shells have been studied by many researchers, which are summarized in the origami models shown in Fig. 2. A typical diamond buckling pattern is shown in Fig. 2(a), in which the hypotenuses of six right triangle elements drawn on a flat paper are connected to make a closed hexagonal cross-section. The shell shown in Fig. 2(b) is obtained by using four obtuse triangle elements, and its cross-section is a square. Its height is much shorter than the former height shown in Fig. 2(a), which is easily collapsed through axial compression. A flat paper with the same folding pattern shown in Fig. 2(b) can be folded completely, shown in Fig. 2(c), before pasting the two ends of the paper. A completely stowed cylindrical shell with a closed polygonal cross-section can be obtained by designing an appropriate obtuse triangle element, which is typically used for the stowed configuration of inflatable tubes, which are deployed through elastic and plastic deformations of the area around the folding lines. Figure 2(d) shows a more general form of buckled cylindrical shells composed of trapezoidal elements, in which four folding lines meet at a single point. In the buckled shell shown in Fig. 2(d), by reversing the zigzag folding lines along the axial direction alternately, another origami model shown in Fig. 2(e) composed of sheared trapezoids is obtained, and its cross-section becomes a star-shaped polygon. It has been reported that inflatable tubes with the star-shaped polygonal folding lines deploy in a more straightforward manner (Senda, et al., 2006). When the sheared trapezoidal shape changes to a parallelogram, the radius of the cylinder becomes infinite, which corresponds to Miura-ori.

\section{Basic consideration in folding flat membranes}

\subsection{Types of folding and one-dimensional deployable membrane structures}

The basis of deployable structures is how to change the distance between two points (Natori, et al., 2008a). Onedimensional structural elements can be changed from fully deployed configuration to stowed configuration in three ways, as shown in Fig. 3: zigzag fold, roll-up, and telescopic fold. Typical deployable membrane space structures that use the zigzag folding concept and roll-up concept are shown in Fig. 4, which are one-dimensional membrane solar cell arrays. A zigzag folding along a straight-line direction is so-called "accordion folding", which can drastically change their length in the deployment direction, and occasionally, this type of folding shows unexpected deformation, such what is shown in Fig. 5, along the width direction. Causes of the elastic deformations, which are shown in Figs. 5(a) and (b), are the lack of stiffness in the support structure and an insufficient design against thermal input, respectively. Reverse folding of flexible elements shown in Fig. 5(c) is possible due to the lack of a hinge-line stiffness along the width direction (Higuchi, 2002), whose deformation pattern is illustrated in Fig. 5(c-2) using an origami image. 


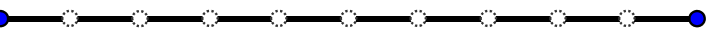

(a) Fully deployed configuration
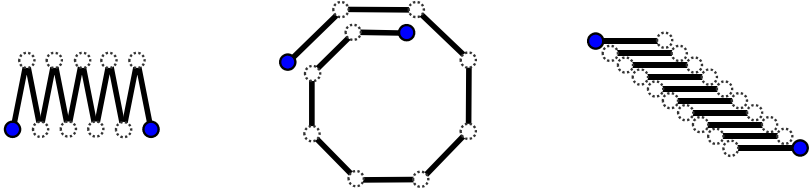

(c) Roll-up

(d) Telescopic fold

(b) Z-fold

Fig. 3 Types of folding
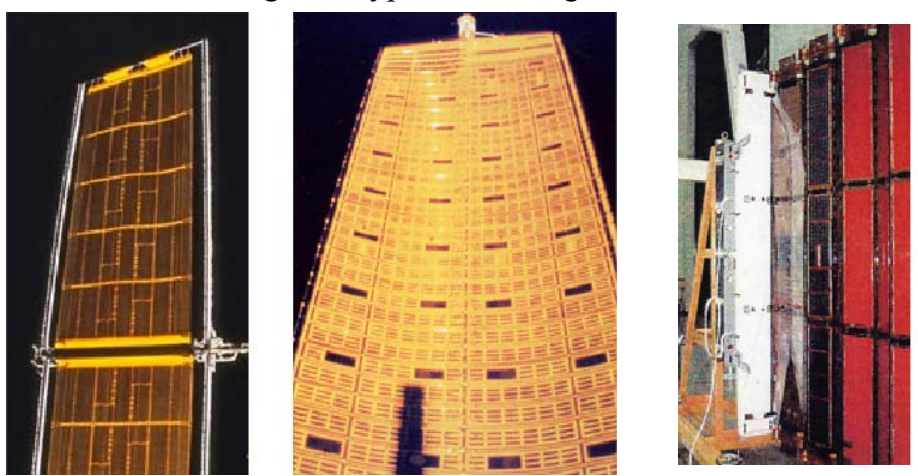

(c1) SFU
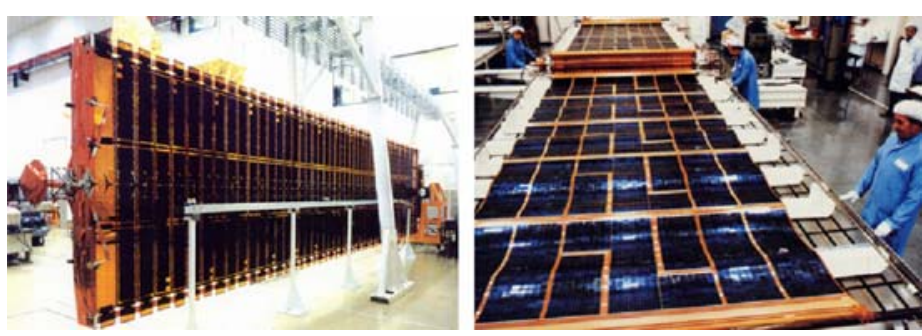

(a) SFU: Space Flyer Unit

(b) HST: Hubble space telescope

Fig. 4 Deployable membrane arrays (One-dimensional)
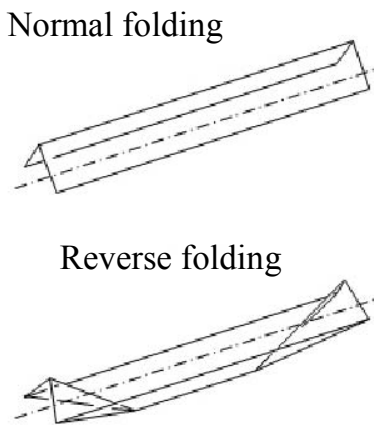

(c2) Origami image

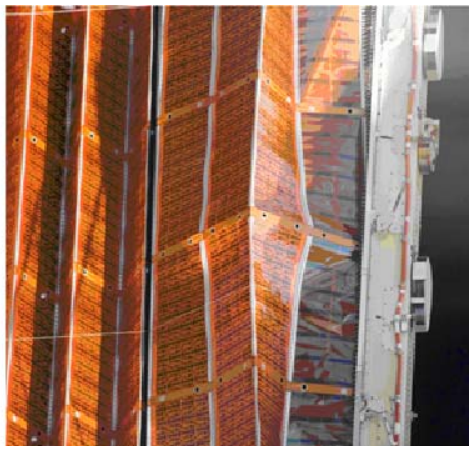

(c) Reverse folding

(a) Twist, HST

(b) Curl, SAFE

Fig. 5 Large deformation of flexible solar cell arrays

HST: Dec. 1999； SAFE: Solar Array Flight Experiment, Sept. 1984； SFU: Jan. 1996； ISS: Dec. 2006

\subsection{Miura-ori and a two-dimensional deployable array}

One-dimensional deployable membrane array structures can deform slightly in their width direction. To apply zigzag folding in both positive orthogonal directions, Miura-ori (Tanizawa and Miura 1978) can be constructed, as shown in Fig. 6(a), which is interpreted as the combination of two orthogonal zigzag folding in a rectangular coordinate system. Its deployment experiment in space as a solar cell array structure was performed, which is shown in Figs. 6(b), (c) and (d), using its 1/4 partial model, and the rather simple retraction of the Miura-ori was confirmed (Natori, et al., 1997) due to the inherent synchronous motions in two orthogonal directions.

\subsection{Planar membrane sail structures and basic folding patterns}

From early 2000, in space structure engineering, light-weight efficient structure systems, called gossamer structure systems, have received much attention; a typical example is a solar sail structure (Greschik and Mikulas, 2002). Figure 7 shows two representative hardware models of sail structures. The four-quadrant sail shown in Fig. 7(a) (Murphy, et al., 2005) is deployed by extendible masts, and the stripe sail shown in Fig. 7(b) (Lichodziejewski, et al., 2005) is deployed by inflatable tubes. Due to the small thickness of the membrane, there has been no special attention given to the folding patterns of the membrane.

Similar to accordion folding defined in the previous section as zigzag folding along a straight-line direction, the wellknown "fan folding" is interpreted as zigzag folding along a curved line. Both ends of fan folding are open, generally as a one-dimensional deployable element. However, when they are connected to each other, it becomes a closed configuration in a two-dimensional polar coordinate system, referred to as "umbrella folding", as shown in Fig. 8(a). Stowing the umbrella along its vertical axis to shorten its height, exactly like a folding umbrella, this folding pattern, which is shown in Fig. 8(b), contains folding lines that correspond to those along the circumferential direction, called the circumferential folding. In umbrella folding, the center part must be sufficiently small to maintain its symmetric properties. However, it is possible to set a circle or polygon at the center, and in this case, the folding lines must be tangential to it, which leads to the tangential line folding shown in Fig. 9. Its folding lines are slightly curved to include the thickness effect of the membrane. In the rectangular coordinate system, elements can move in both the positive and negative directions of its coordinate axis. Therefore, in Miura-ori, the thickness effect appears only as local elastic 


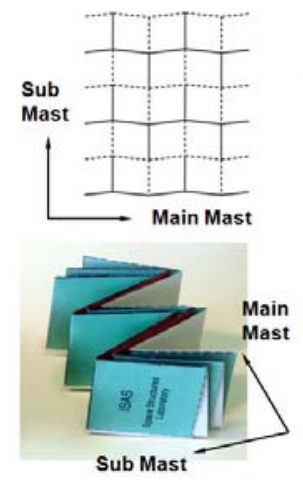

(a) Miura-ori

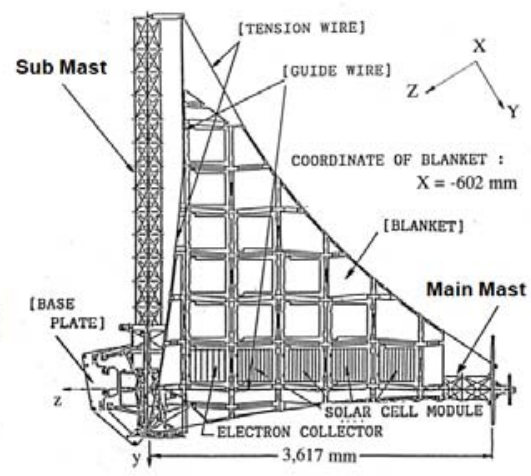

(b) $2 \mathrm{D}$ array (1/4 model)

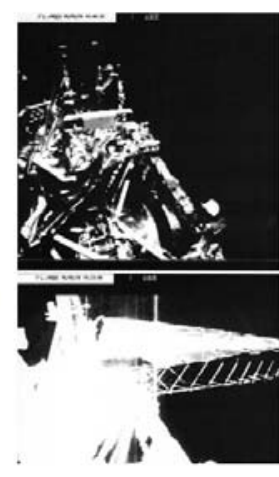

(c) $2 \mathrm{D}$ array in orbit

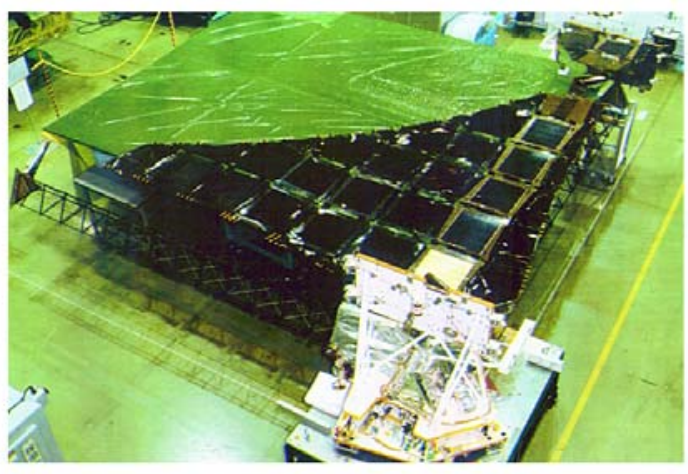

(d) 2D array after retrieval

Fig. 6 Two-dimensional (2D) deployable array onboard SFU

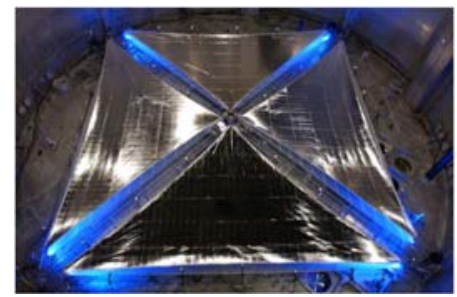

(a) Four-quadrant design

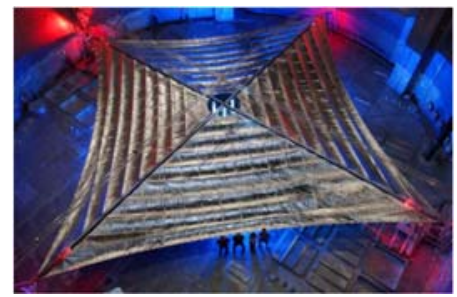

(b) Stripe design

Fig. 7 Recent solar sail structures

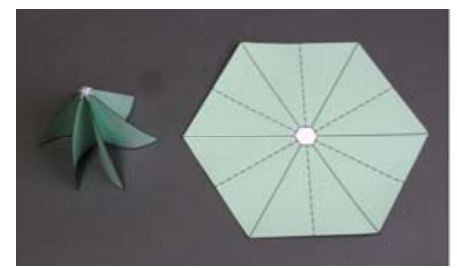

(a) Basic umbrella folding

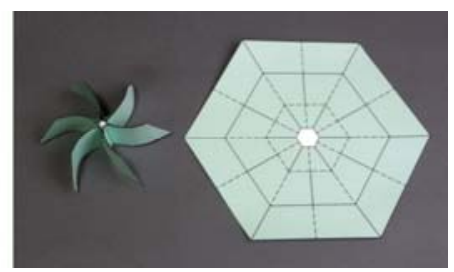

(b) Circumferential folding

Fig. 8 Umbrella folding

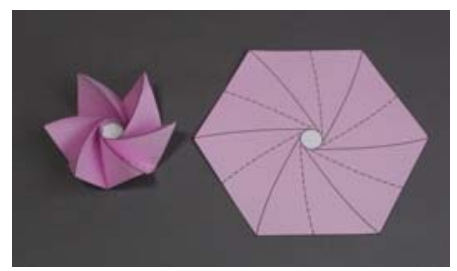

Fig. 9 Tangential line folding

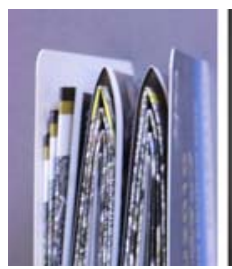

(a) Miura-ori

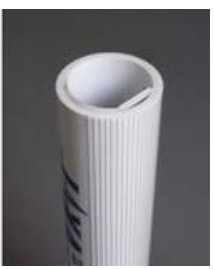

(b) Calendar

Fig. 10 Thickness effect

deformation around the corner parts, which is shown in Fig. 10(a), and it is unnecessary to change its straight folding lines. In contrast, in the polar coordinate system, elements cannot move in their negative region beyond zero, and the thickness effect appears more remarkably throughout the entire structure as, for example, the difference in the circumferential length, as shown in Fig. 10(b) (this might be called a "calendar effect"). For future large gossamer systems, such as large solar cell arrays, phased antenna arrays, etc., the thickness of the membrane elements might be one or two orders larger compared with the thin membrane for solar sail structures, and thus, it becomes important to consider its effect.

As one solution to include the thickness effect, the curved folding lines shown in Fig. 9 can be used, which gradually deviate from straight lines to form spiral lines (Natori, et al., 2007b). Adding additional folding lines to reduce the stowed height of the tangential line folding shown in Fig. 9, a spiral folding pattern, as shown in Fig. 11(a), can be obtained (Natori, et al., 2010). In the figure, the shaded strip bounded by folding lines located along the radial direction is wrapped around a center body and bent at each corner of the body. It is interpreted that the spiral folding is obtained from the 


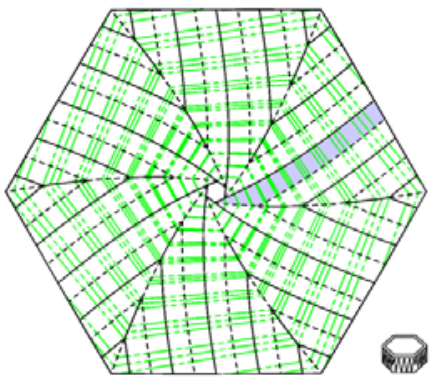

(a) Spiral folding

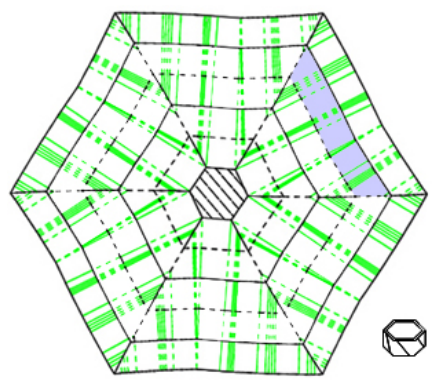

(b) Circumferential folding

Fig. 11 Two representative folding patterns of a flat membrane surface Solid line: mountain folding, Dashed line: valley folding, Thin line: line bent along the center body

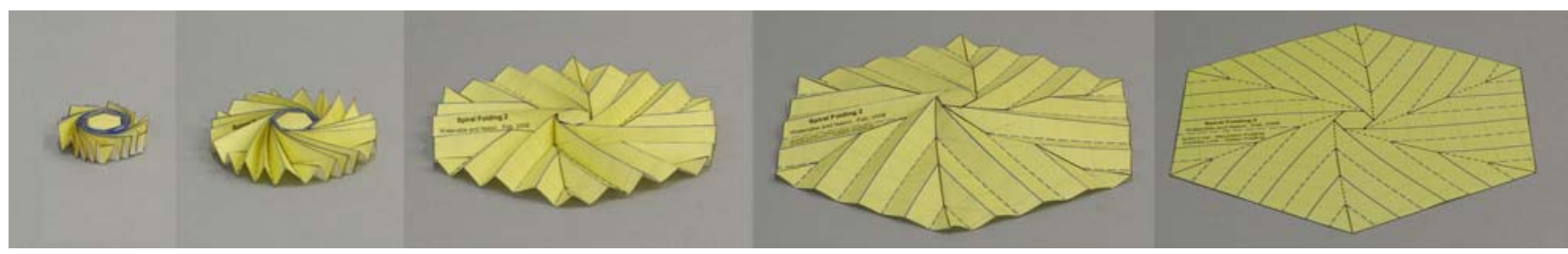

(a) Spiral folding

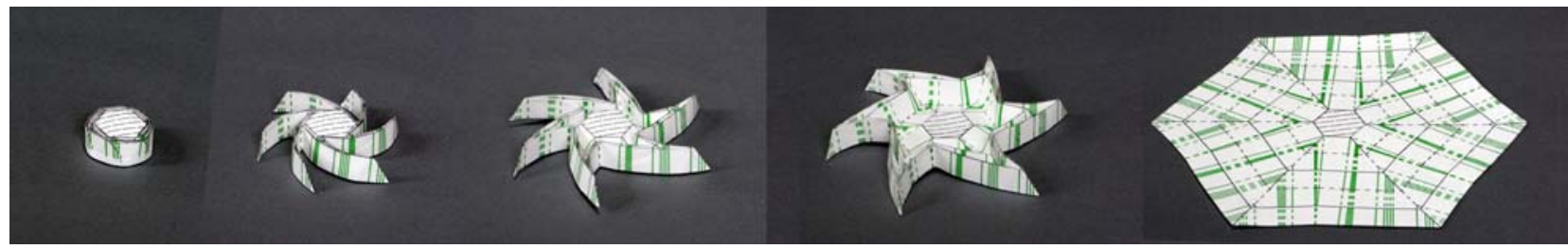

(b) Circumferential folding

Fig. 12 Deployment sequence of disk-like origami models

zigzag folding along the circumferential direction and from the roll-up folding along the radial direction in the polar coordinate system. The width of the strips is essentially constant to avoid a singular problem caused by the concentration of folding lines near the center body. Folding lines are slightly curved and consist of piecewise straight-line segments with the length decided along the external form of the center polygon. The lines become straight when the thickness effect can be neglected. Considering a center body at the center in the circumferential folding shown in Fig. 8(b), its detailed folding pattern shown in Fig. 11(b) is obtained. The shaded strip located along the circumferential direction is wrapped around a center body and bent at each corner. Thus, the circumferential folding can be obtained from the zigzag folding along the radial direction and the roll-up folding along the circumferential direction. Folding lines are also slightly curved to include the thickness effect.

Deployment of the spiral folding can be accomplished smoothly simply through releasing the wrapped strips continuously, as shown using an origami model in Fig. 12(a). Deployment of the circumferential folding is also shown in Fig. 12(b). Initially, the arms are deployed (first deployment phase), followed by full two-dimensional deployment (second deployment phase). An actual flight experiment (Sawada, et al., 2011) using a circumferentially folded membrane, whose system-side design was developed earlier at that time, was performed in 2010, which was a spin-type solar sail without any booms. Its details are shown in Fig. 13, which include an actual quadrant membrane, images taken in space by onboard side-view cameras and separation cameras, and the planned deployment sequence. Many of the folding lines of the membrane were approximated to be straight lines for simplicity, and difficulty arose in stowing four arms due to their calendar effects, which were forced to be stowed.

\subsection{Modular space structure systems and their origami models}

The spiral folding pattern maintains good symmetrical properties, and various geometrical extensions are possible. 


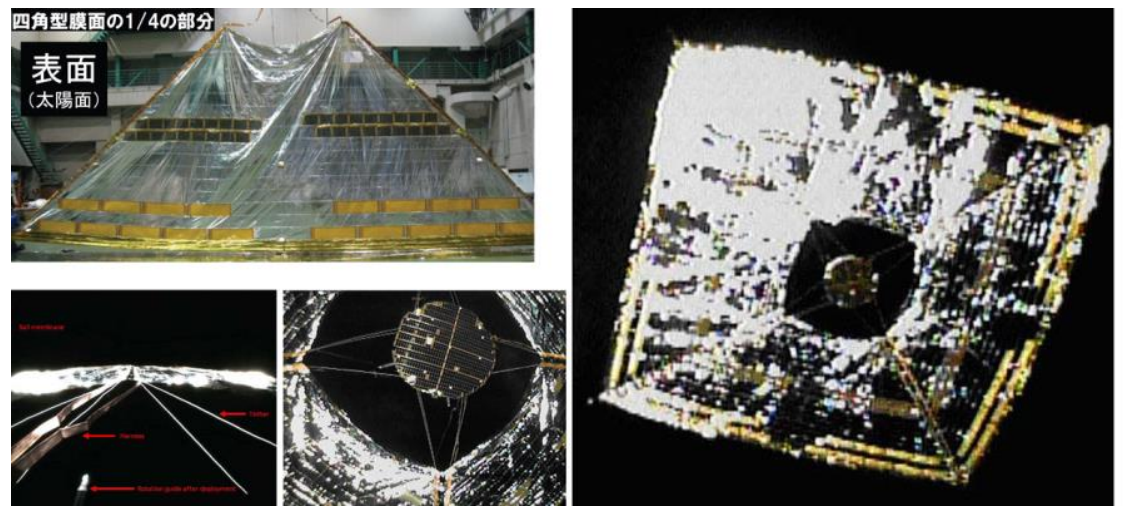

(a) Membrane and pictures taken in space

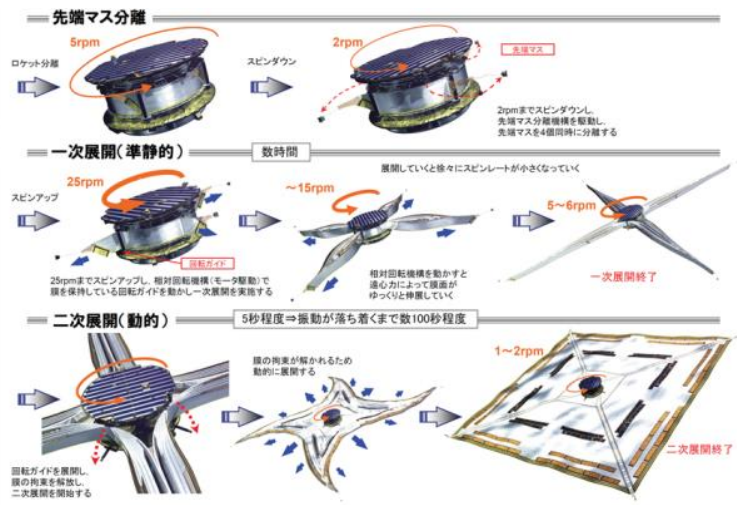

(b) Deployment sequence

Fig. 13 Solar sail demonstrator "IKAROS"

Actual example of a solar sail structure with circumferential folding pattern

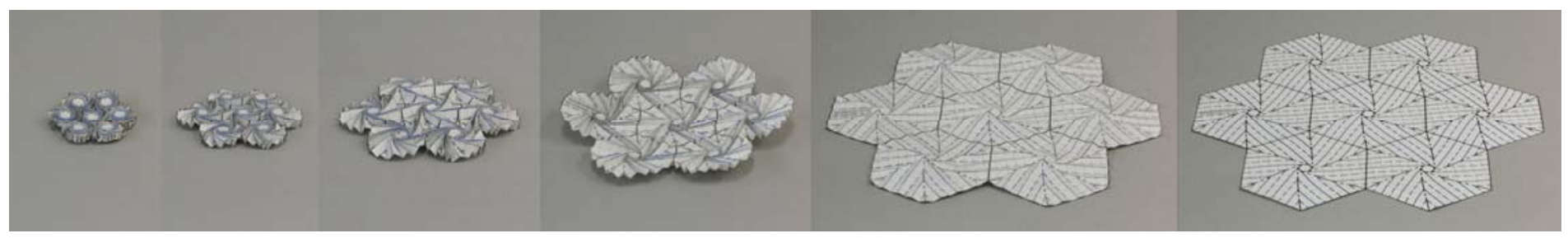

Fig. 14 Origami model of a deployable modular space structure system

It is well known in geometry that a regular hexagon can tessellate a planar surface without any opening. Regular hexagonal pattern with spiral folding also can tessellate a planar surface, which means that a planar surface can be folded around many center bodies distributed regularly on the surface, such as that shown in Fig. 14 using an origami model. Because small paper origami models are strongly affected by locally reinforced stiffness due to folded lines, some intermediate configurations shown in Fig. 14 might be different from those of the actual hardware; however, it is shown that the concept of deployable modular structure systems would have some possibility. It should be noted that a fully tessellated pattern corresponds to a special case of hierarchical modular patterns (Kishimoto and Natori, 2006) and that introducing deployable membrane elements to hierarchical modular structure systems will lead to proper designs for future large-scale space systems. To realize more advanced space structure systems, availability of inflatable tubes or deployable booms with sufficient rigid properties must be carefully considered. In Fig. 14, if inflatable tubes are embedded along the hexagonal peripheral edges as deployment actuators and supporting members after deployment, they must be stowed in zigzag folding, and the modified zigzag folding pattern shown in the previous section (Figs. 1(b) and (c)) would be a good candidate for this purpose. Also note that modules with circumferential folding shown in Fig. 11(b) cannot tessellate a surface due to the lack of symmetry.

Up until here, hexagonal modules have been treated; however, similar considerations can be applied to other basic polygonal modules, and Figure 15 shows a triangular module with spiral folding. Furthermore, folding of threedimensional framework structures covered by a membrane could be possible, such as, for example, a fullerene-shaped structure, in which both hexagonal and pentagonal modules with spirally folded membrane are used (Natori, et al., 2008b).

\subsection{Dual relation between spiral folding and circumferential folding and dual modules}

Because one tessellated flat surface easily introduces a dual surface, a flat surface tessellated by hexagonal modules with spiral folding can also be interpreted as a surface tessellated by triangular modules, as shown in Fig. 16. Figure 17(a) shows this triangular module. Its inside folding pattern corresponds to circumferential folding, where the membrane is folded around three vertices. In this case, rolled-up booms can be set along its peripheral edges. A compound module composed of six triangular modules shown in Fig. 17(b) could have both peripheral and rib booms, and its origami model corresponds to an inside part of that shown in Fig. 14. This module might be appropriate as structural components for efficient space structure systems, which is now under development using bi-convex booms covered by a braid (Watanabe, et al., 2012).

The aforementioned surface is the tessellation of a hexagonal module, and conversely, a regular triangular module 


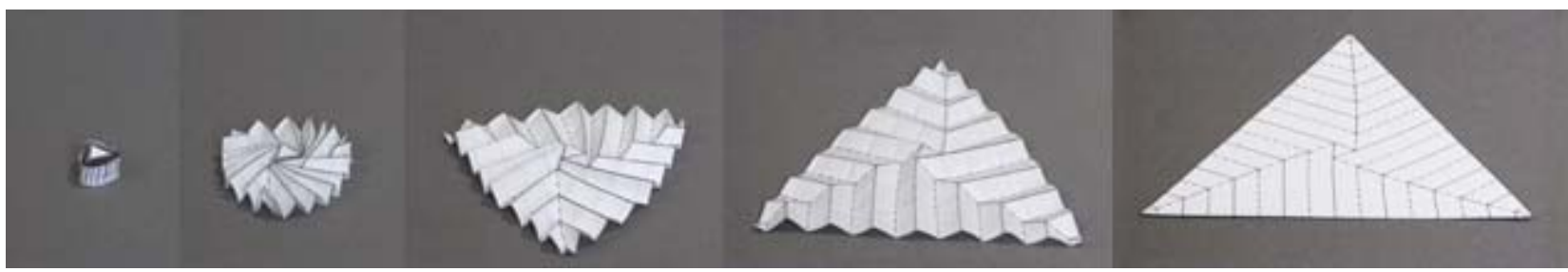

Fig. 15 Origami model of triangular module with spiral folding

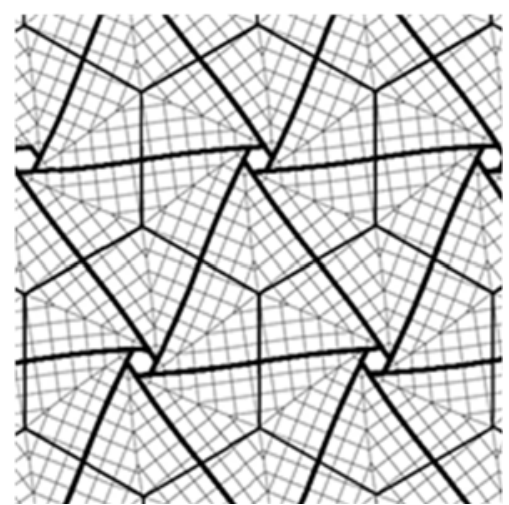

Fig. 16 Tessellation of a planar surface using hexagonal modules with regular spiral folding and their dual surface
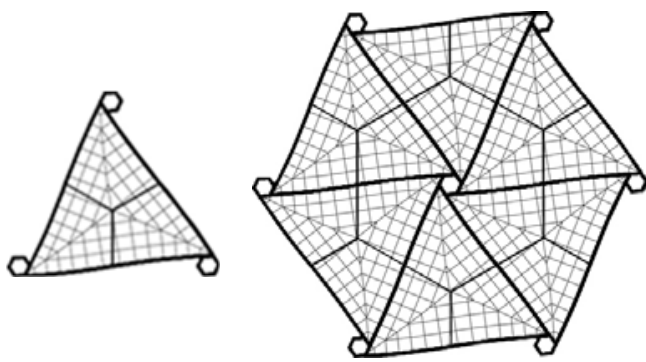

(a) Dual triangular module

(b) Dual hexagonal compound module composed of six dual triangular modules

Fig. 17 Membrane module driven from dual tessellation of hexagonal modules with regular spiral folding pattern

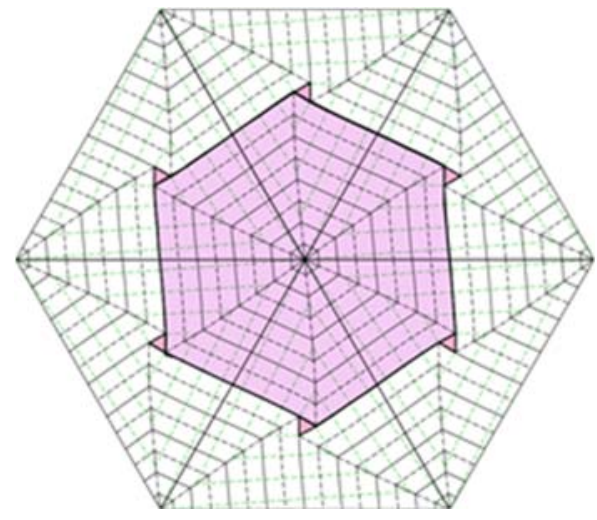

Fig.18 Tessellation pattern of triangular modules with spiral folding and their dual module

with spiral folding (Fig. 15) can also tessellate a planar surface, as shown in Fig. 18. Its dual hexagonal module is also shown shaded in the figure, which tessellates the same surface, and is also stowed through circumferential folding wrapped around each vertex.

It is well known that a dual pattern of a tessellated surface by squares is also the same, and the tessellated surface by square modules with spiral folding, and the outward forms of the corresponding dual square modules are shown in Fig. 19. The stowing process of the square modules is shown in Fig. 20 using origami models. It is noted again that several intermediate configurations shown in these origami models might be different from actual hardware due to reinforced stiffness around the folded lines; however, the differences in their deployment aspects among those modules are clearly shown.

\subsection{Membrane structures actuated and supported by deployable booms}

A membrane is essentially a tension element, and for easy treatment of membrane structures, compound structures composed of membranes and booms are desirable. Various types of deployable booms, such as coilable lattice booms, inflatable rigidized booms, shape memory composite booms, etc., are considered to be useful. Recently, among many of them, convex tape booms and their versions have received much attention because convex tapes can be easily rolled up under their flattened deformation, and from this deformed configuration, they can also be easily deployed by themselves through the release of stored elastic energy (Banik and Murphey, 2008). Because a membrane with spiral folding pattern is folded around the center body, rolled-up convex booms are compatible with the folding pattern to create an efficient membrane structure. For that purpose, several peripheral parts of the original regular spiral folding pattern are cut off, as shown in Fig. 21(a), and along its diagonal lines, convex booms are arranged, which are attached to the membrane at several appropriate points or embedded into the membrane, if possible. Because they are considered to be stowed by winding around the center body together with membranes in a flattened cross-sectional configuration and their thickness is considerably thick compared with the membrane thickness, the folding lines shown in Fig. 21 are not necessarily strictly curved lines. Several alternate engineering solutions to the spiral folding pattern have also been studied (Sakamoto, et al., 2012). To realize reliable deployment, the level of stowed elastic energy must be appropriately high, 


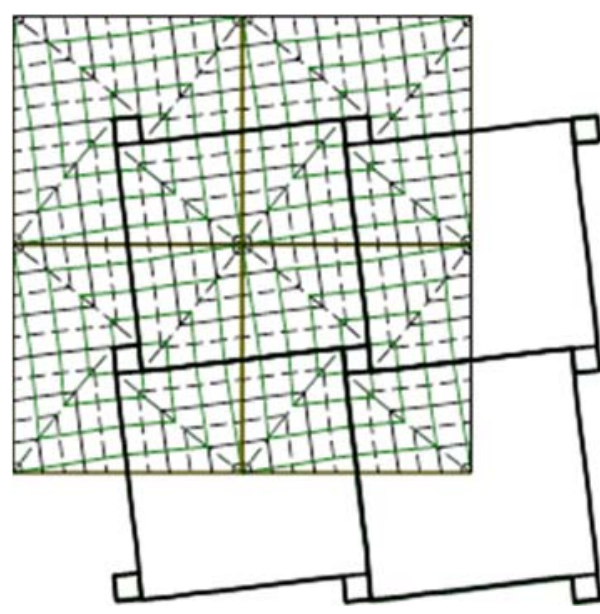

Fig. 19 Tessellation pattern of square modules and their dual modules

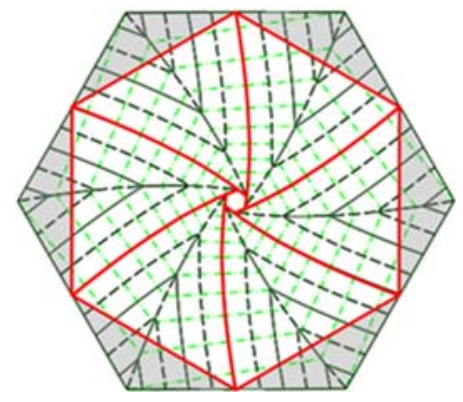

(a) Modified spiral folding pattern

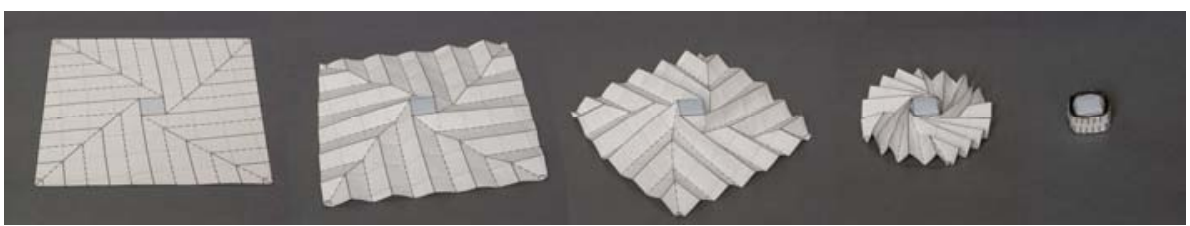

(a) Square module with spiral folding

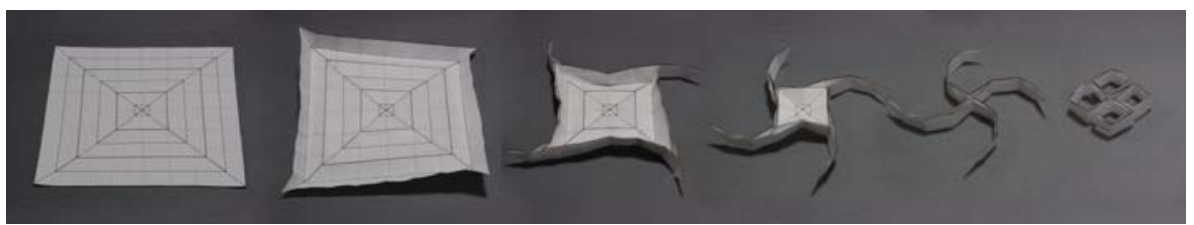

(b) Dual square module

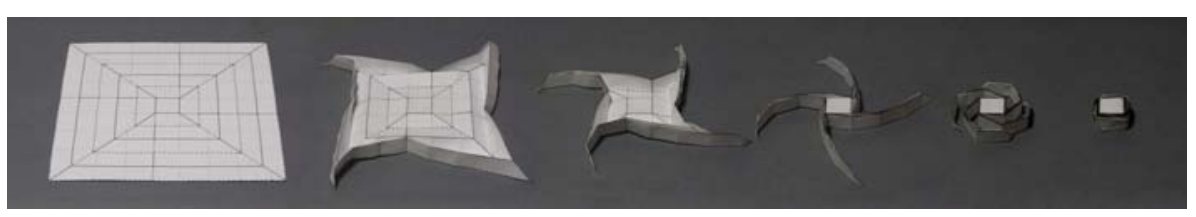

(c) Square module with circumferential folding

Fig. 20 Stowing process of square modules

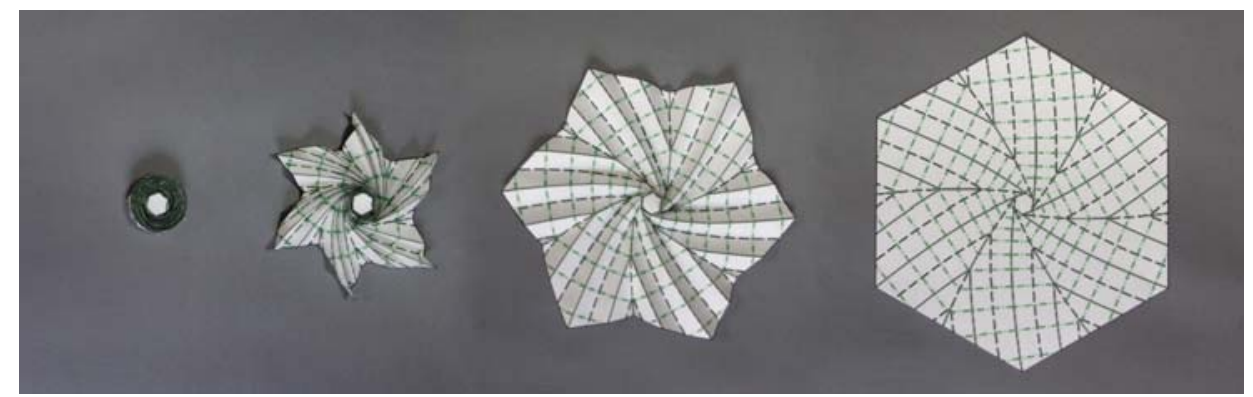

(b) Deployment sequence of an origami model with modified spiral folding

Fig. 21 Version of spiral folding pattern suitable for rolled-up rib booms and its origami model which typically causes impulsive deployment. To reduce such impulsive effects, the stepwise deployment procedure has been introduced as the simplest design of a compound membrane. Booms and membranes are fixed to the center body at several fixed points using an appropriate fixing mechanisms and are released one after the other, which is defined as stepwise deployment (Natori, et al. 2012). Several deployment aspects of small-scale models are shown in Fig. 22, and a successful three-step deployment is clearly shown.

\section{Examples of deployable concepts in nature}

\subsection{Plant leaves and flowers and the limitation of origami models}

There are two types of morphological changes in objects in nature: their growth phase and their function phase (Natori, et al., 2006), (Natori, et al., 2011). As an example, the difference between these changes in the leaves of Mimosa Pudica is shown in Fig. 23. Their change of configurations in response to their growth is shown in Fig. 23(a). In their function phase after reaching full growth, they extensively change their response to physical stimulant, as shown in Fig. 23(b), and their origami model is much simpler because they also compound their wing leaves. After the growth phase, their changes are irreversible, though changes are reversible during the function phase. Because an origami model presents a deformed configuration change, it can effectively demonstrate the morphological changes of objects in nature during their function phase, though cannot exactly explain the changes during their growth phase. This fact must be considered, particularly when developing novel aerospace applications inspired by objects in nature through origami models. For example, the change of simple plant leaves from their early closed shape to fully opened shape, as shown in Fig. 24(a), seems to be explained by origami models shown in Fig. 24(b) (Kresling, 1994). However their fully opened shape is formed on the formation basis of veins, and the detailed shape around the veins is markedly different from the 


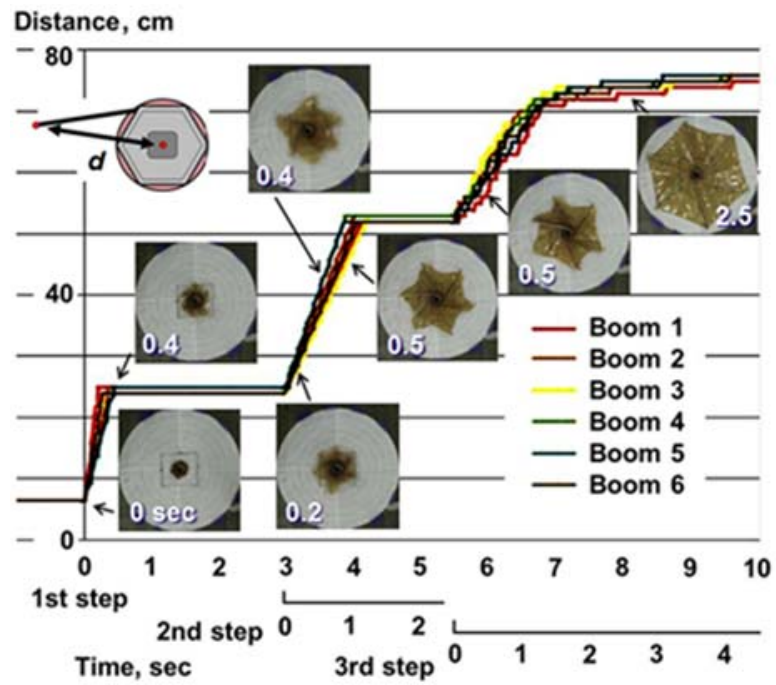

Fig. 22 Deployment behavior of membrane structure model actuated by convex tape booms

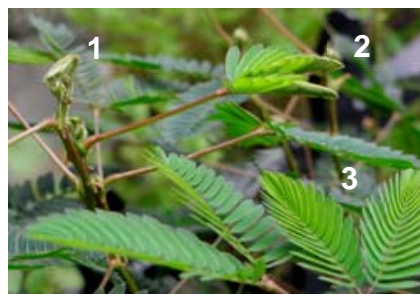

(a) Change due to growth
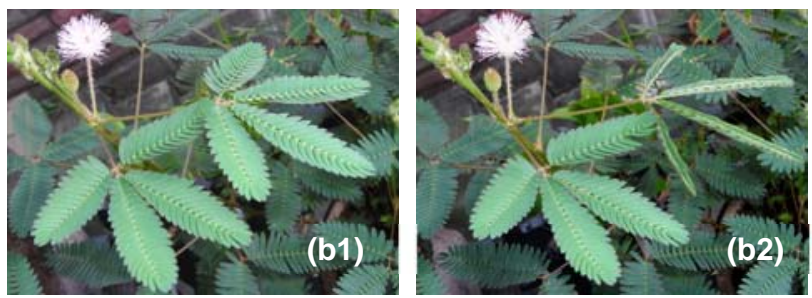

(b) Change against physical stimulant

Fig. 23 Morphological change of leaves of a sensitive plant (Mimosa Pudica)

origami model. In the origami model, the centerline, which is where two slanted waves join, is bent in the out-of-plane direction; however, the main vein shown in Fig. 23(a) is bent in the in-plane direction. Considerations of this type of difference would likely affect appropriate location of actuators for actual applications.

Figure 25 shows configuration changes during blooming of various types of flowers. A dandelion or sunflower is an aggregation of tubular and ligulate flowers. In the intermediate configuration of a dandelion just before full blooming, two ligulate flower shapes with different lengths appear, as shown in Fig. 25(a), which grow abruptly from a shorter shape to a longer shape. Ligulate flowers of a sunflower deploy sequentially from the stowed shape, as shown in Fig. 25(b), which can be expressed using origami models. Logarithmic spiral formation of inside tubular flowers is also shown in Fig. 25(b2), which simultaneously grows. Broad petals from a poppy are crumpled without any regular folding patterns; however, after their deployment, they form a fine curved surface, as shown in Fig. 25(c), which is perhaps beyond origami modeling. Bindweed (convolvulus) and morning glory flowers are typical examples of infundibular flowers, as shown in Fig. 26, whose configuration is different from that shown in Fig. 25. The florescence of bindweed is shown in Fig. 26(a), and the circular petal opens from the stowed configuration convoluted inside. Buds of bindweed and morning glory flowers at the beginning of their florescence are shown in Fig. 26(b). It is shown that the bud configuration of a bindweed flower is almost straight, whereas that of a morning glory is wound helically. The fully opened starfish shape of the bindweed is more regular compared with that of the morning glory, as shown in Fig. 26. Bindweed opens during the day and closes at night for a few days, whereas a morning glory blooms only once in the morning, which means a regular shape is convenient for opening and closing motions. Figure 27 shows the origami model of a bindweed. Five fan-shaped parts of a circular petal are convoluted inside, which is the opposite way from the outside winding of the umbrella folding shown previously (Fig. 8(a)), and the resulting folded configuration is a pentagonal pyramid, whose outward part corresponds to the starfish shape. It is rather difficult to complete an inside convolution in an origami model compared with an outside winding, which means its opening process is irreversible. 


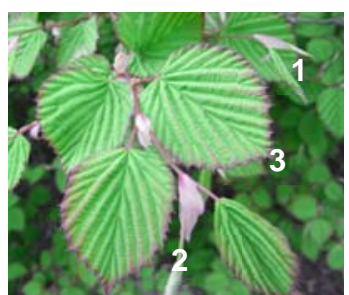

(a) Shape change of plant leaves

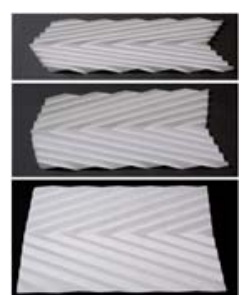

(b) Origami model

Fig. 24 Shape change during growth phase

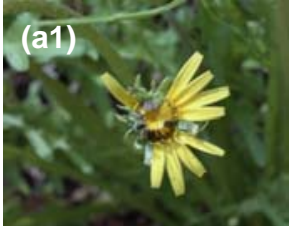

(a) Dandelion

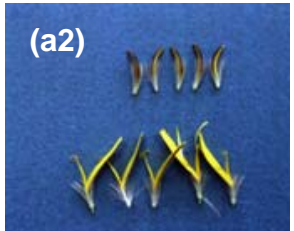

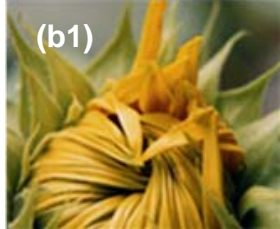

(b) Sunflower

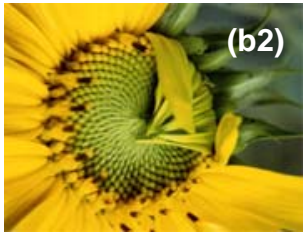

Fig. 25 Blooming of various types of flowers

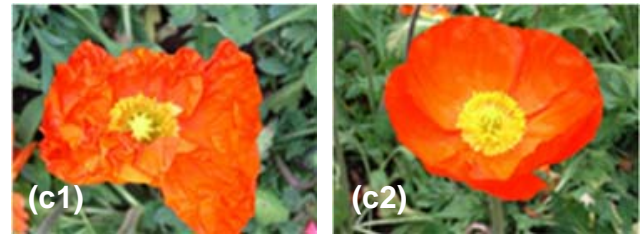

(c) Poppy
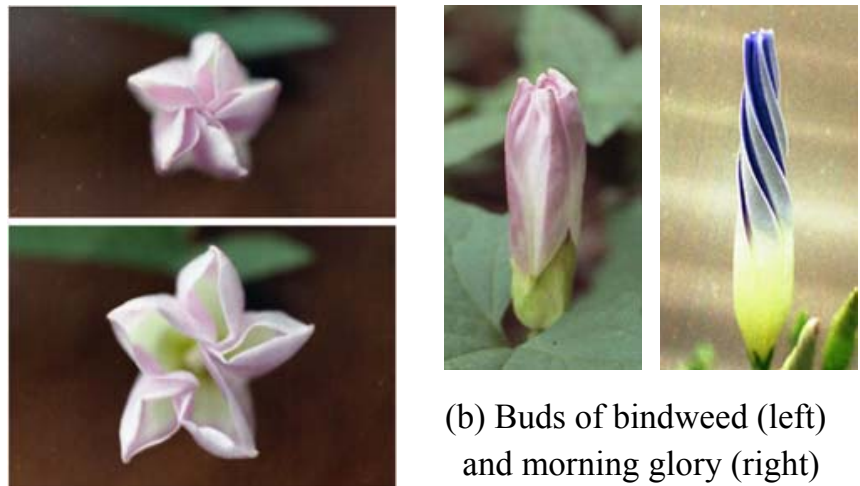

(b) Buds of bindweed (left) and morning glory (right)

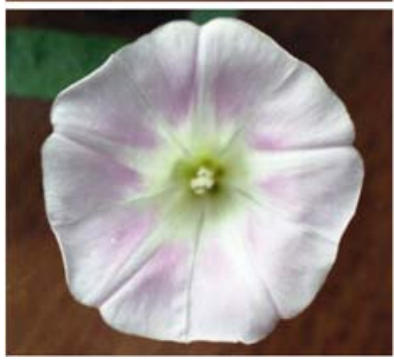

(a) Florescence of a bindweed

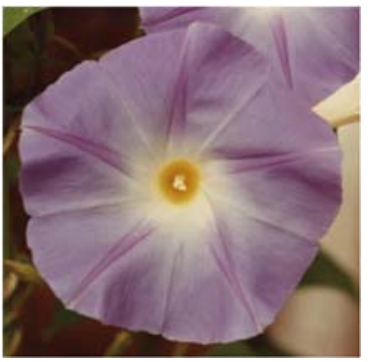

(c) Morning glory

Fig. 26 Bindweed and morning glory 


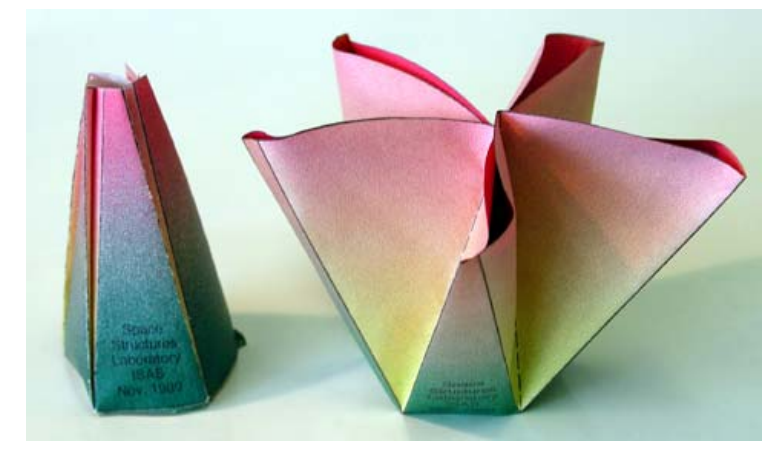

Fig. 27 Origami model of a bindweed
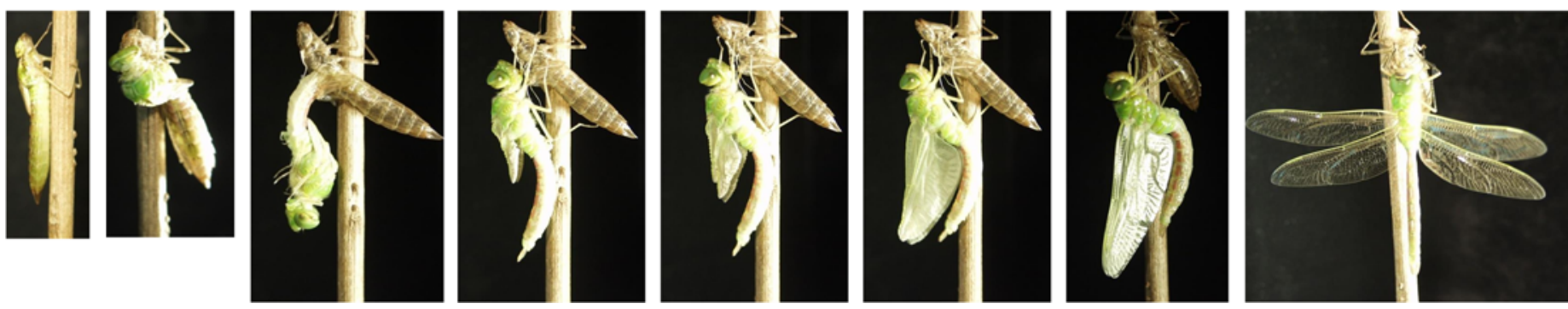

Fig. 28 Eclosion process of a dragonfly

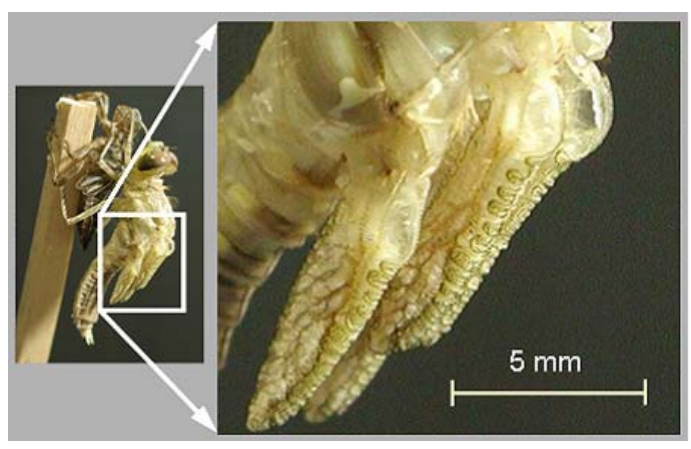

(a) Folded inflatable tubes

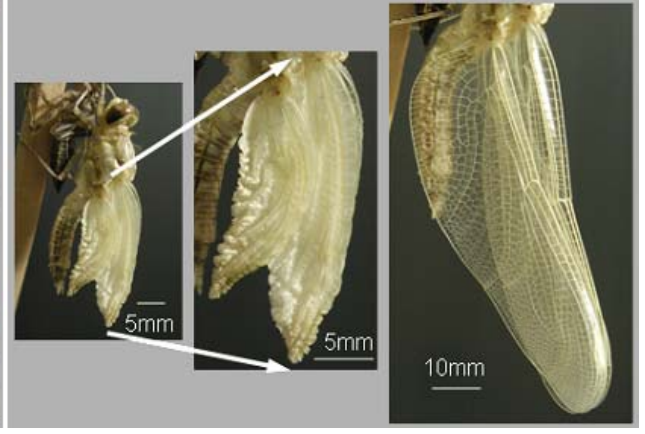

(b) Inflatable tube network

Fig. 29 Details of dragonfly wing during eclosion

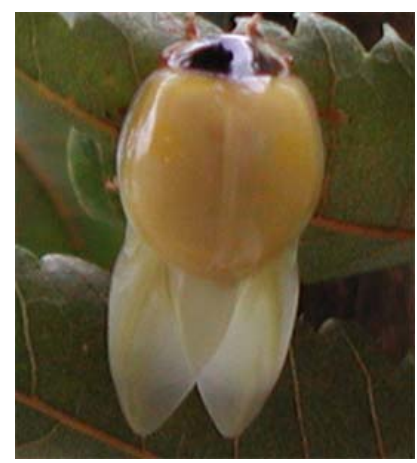

Fig. 30 Lady bug immediately following eclosion

\subsection{Eclosion of dragonflies and their inflatable tube networks}

Morphological changes during eclosion of insects occur during their growth phase, and are essentially irreversible. These changes are not directly appropriate for origami modeling; however, there are many suggestions for their usefulness in better aerospace applications.

Figure 28 shows the eclosion process of a dragonfly. After removing the shell during its larva period, he waits in the upside-down position for a period of time to gain rigidity in the legs. Then, he changes his posture as if to fit the direction of wing expansion to the gravity direction. Wings expand due to the flux of body fluid sent from his chest through the vein network. Changes in body color indicate the progress in creating a more rigid outer shell. After rigidization is complete, he opens his wings and flies away. Figure 29 shows the details just before and during their expansion. Four thin membrane wings are stowed in four sheaths on the back of a larva of a dragonfly. Through the detailed investigation of dragonflies' hind wings in their inside sheaths of last instar larvas through microscope observation, it was observed that their membranes and veins are hierarchically folded based on zigzag folding with approximately forty folds, which is highly efficient packing. It might be that hierarchical folding patterns are naturally generated because the growth of their membranes is within a limited space of their sheaths. Butterflies and cicadas show similar eclosion processes (Natori, et al., 2006). 


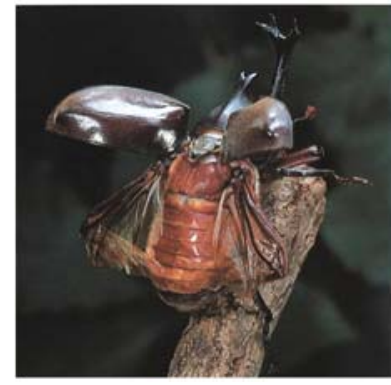

(a) Just before flight

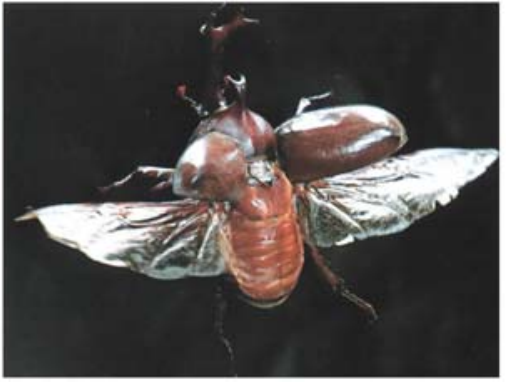

(b) Fully deployed

Fig. 31 Fore and hind wings of a Japanese rhinoceros beetle *

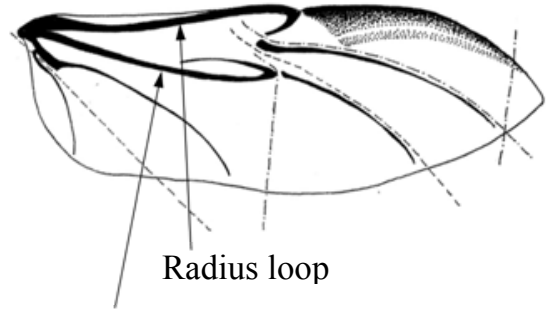

Medial loop

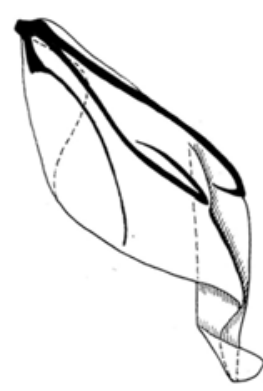

(a) Folding process of a Japanese rhinoceros beetle ${ }^{\text {a }}$

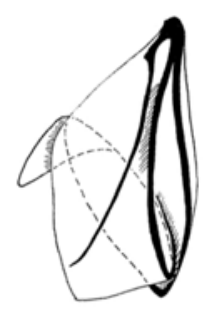

(b) Folding lines of a pselaphine beetle (Nomura, 1997)

Fig. 32 Folding of beetle's hind wings

Figure 30 shows the wings of a lady bug just after eclosion, and their folding pattern is rather simple compared with that of dragonflies. Beetles (coleopterous insects) show their priority for protecting hind-wings, and their conspicuous morphological change of wings is for deployment and stowage motion during their function phase, which can be expressed by origami models.

\subsection{Folding patterns of beetles' hind wing}

Insect wings essentially transform from the walls of middle and hind thoraxes and the inside veins, which had been tracheas before, and are wrapped by sack-like (balloon) membranes. Moreover, in beetles, fore wings change into elytra, and hind wings have folding mechanisms for stowage (Natori, et al., 2011). Figure 31 shows the wing deployment of a large beetle for flight, whose body length is approximately $60 \mathrm{~mm}$. Its folding mechanism is illustrated in Fig. 32(a) ${ }^{\mathrm{a}}$ with folding lines. The motion of two major veins, called the radius and medial loops, actuates folding, which is easily modeled by origami. The smaller the size of beetles becomes, the greater the number of folds increases, which reaches about 10 along span direction in an extreme case. One example of the folding patterns of a small beetles' hind wing is shown in Fig. 32(b), whose body length is approximately $2 \mathrm{~mm}$ (Nomura, 1997). Because of being small and consequently, sufficiently light-weight, beetles can easily fly or float in air. The two major veins are through the cooperative motions of the veins, fore wings and abdomen, and the fore wings are fitted to the abdomen at several points to keep the stowed hind wings inside.

\section{Conclusions}

The study showed how conceptual origami models have contributed to the design of various deployable space structures and what can be learned from nature by keeping the origami concept in mind. Several basic design considerations to fit the concepts to actual mechanical hardware are clarified.

a Unpublished figure given by Dr. S. Nomura (National Museum of Nature and Science, Tokyo, Japan), cited in (Natori, et al., 2011). 
For one-dimensional inflatable tube structures, smooth deployment of a boom folded in the modified zigzag pattern developed from an origami image is shown, and several origami models of axially buckled cylindrical shell elements related to stowed configurations of inflatable tubes are introduced. It is pointed out that in actual structures, elastic deformation of the tube membrane completes their deployment, in addition to inextensional transformation expressed by origami models.

For planar two-dimensional surface origami models, systematic interpretation of folding patterns is clearly shown by considering their coordinate systems (rectangular or polar) and folding manners (zigzag or roll-up). Considering the thickness effects of disk-like flat surfaces, two typical folding patterns, namely the spiral folding pattern and circumferential folding pattern, are introduced, and their mutual relations through geometrical tessellation are explained. Many model versions, including those driven from their dual properties, are introduced, and a model of deployable modular structures for future large space structure systems is also presented.

Finally, regarding effective gossamer space structure designs, several examples of deployable concepts in nature are introduced, and their interpretation for structural mechanics through origami models is shown. Through the observation of morphological changes of leaves, flowers and insects, it is explained that in objects in nature, there are two types of changes, namely changes during the growth phase and changes during their function phase. It is pointed out that origami models are effective to help understand their mechanisms; however, origami models have several limitation for use in actual applications inspired by them because the changes, particularly during their growth phase, include extensional deformation and irreversible transformation, which might be difficult for present origami materials.

\section{Acknowledgement}

The authors would like to acknowledge Dr. S. Nomura (National Museum of Nature and Science, Japan) for his support for this study.

\section{References}

Banik, J. A. and Murphey, T. W., Synchronous deployed solar sail concept demonstration, 49th AIAA/ASME/ASCE/ AHS/ASC Structures, Structural Dynamics, and Materials Conf., Schaumburg, Illinois (2008), AIAA $2008-2213$.

Freeland, R. E., Bilyeu, G. D., Veal, G. R., Steiner, M. D. and Carson, D. E., Large inflatable deployable antenna flight experiment results, 48th Int'1. Astronautical Cong., Turin, Italy (1997), IAF-97-I.3.01.

Greschik, G. and Mikulas, M. M., Design study of a square solar sail architecture, J. Spacecraft and Rockets, 39 (2002), pp. 653-661.

Higuchi, K., Natori, M. C. and Abe, M., Unexpected behavior of a flexible solar array at retraction under microgravity, Acta Astronautica, Vol.50, No.11 (2002), pp. 681-689.

Jenkins, C. H. (Ed.), Recent advances in gossamer spacecraft (2006), AIAA.

Katsumata, N., Natori, M. C. and Yamakawa, H., Dynamic behavior analysis of inflatable booms in zigzag and modified zigzag patterns, 63rd Int'l. Astronautical Cong., Naples, Italy (2012), IAC-12-C2.3.11.

Kishimoto, N. and Natori, M. C., Hierarchical modular structures and their geometrical configurations for future large space systems, J. Int'l. Assoc. Shell and Spatial Structures (IASS), Vol.47, No.3 (2006), pp.303-309.

Kresling, B., Folded and Unfolded Nature, Origami Science and Art (ed. K. Miura et al), Proc. 2nd Int'l. Meeting of Origami Science and Scientific Origami, Seian Univ. Art and Design, Otsu, Shiga, Japan (1994), pp.93-108.

Lichodziejewski, D., Derbès, B., Slade, K. and Mann, T., Vacuum deployment and testing of a 4-quadrant scalable inflatable rigidizable solar sail system, 46th AIAA/ASME/ASCE/AHS/ASC Structures, Structural Dynamics and Materials Conf., Austin, Texas (2005), AIAA 2005-2122.

Murphy, D. M., McEachen, M. E., Macy, B. D. and Gaspar, G. L., Demonstration of a 20-m solar sail system, 46th AIAA/ ASME/ASCE/AHS/ASC Structures, Structural Dynamics and Materials Conf., Austin, Texas (2005), AIAA 20052126.

Natori, M. C., Kuninaka, H., Higuchi, K. and Onodera, T., In-orbit deployment and post flight analysis of the 2D array on-board SFU, 48th Int'l. Astronautical Congress, Turin, Italy (1997), IAF-97-I.1.01.

Natori, M. C., Kishimoto, N., Ukegawa, K. and Higuch, K., Concept of morphological changes in bio-systems and artifacts, 3rd Int'l. Symp. Intelligent Artifacts and Bio-Systems, Daejeon, Korea (2006).

Natori, M. C., Concepts of advanced light space structure systems and their characteristics, Japanese 49th Conf. Structures and Materials (Kozo-Kyodo), JSASS/JSME, Fukushima, Japan (2007a), pp. 1-6 (in English). 
Natori1, M. C., Watanabe, H., Kishimoto, N. and Higuchi, K., Folding patterns of deployable membrane space structures considering their thickness effects, 18th Int'l. Conf. Adaptive Structures and Technologies (ICAST), Ottawa, Canada (2007b).

Natori, M. C., Kishimoto, N., Higuchi, K. and Yamakawa, H., Basic geometrical consideration on deployable and adaptive structures for efficient spacecraft systems, 26th Int'l. Symp. Space Technology and Science (ISTS), Hamamatsu, Japan (2008a), ISTS 2008-c-05.

Natori, M. C., Yamakawa, H., Kishimoto, N. and Higuchi, K., Concept of large space structure systems using deployable membrane modules embedded with inflatable tubes, 19th Int'l. Conf. Adaptive Structures and Technologies (ICAST), Ascona, Switzerland (2008b), pp. 610-615.

Natori, M. C., Katsumata, N. and Yamakawa, H., Membrane modular space structure systems and deployment characteristics of their inflatable tube elements, 51st AIAA/ASME/ASCE/ AHS/ASC Structures, Structural Dynamics, and Materials (SDM) Conf., Orlando, FL (2010), AIAA 2010-2909.

Natori, M. C., Kishimoto, N., Nomura, S. and Yamakawa, H., Considerations on morphological changes of objects in nature and artifacts," Proc. Japanese 53rd Conf. Structures and Materials (Kozo-Kyodo), Akita, Japan (2011) (in Japanese).

Natori, M. C., Hori, H., Sawai, K., Okuizumi, N. and Yamakawa, H., Stepwise deployment of membrane space structures rolled-up together with support booms, 63rd Int'l. Astronautical Congress, Naples, Italy (2012), IAC-12-C2.2.9.

Nomura, S., Flight of pselaphine beetles - morphological review on flight mechanism of tiny beetles, Insectarium, Vol.34 (1997), pp.22-30 (in Japanese).

Sakamoto, H., Natori, M. C., Kadonishi1, S. and Okuma, M., Folding Patterns of Planar Gossamer Space Structures Consisting of Membranes and Booms, 23rd Int'l. Conf. Adaptive Structures and Technologies, Nanjing, China (2012), ICAST2012 \#63.

Sawada, H., Mori, O., Okuizumi, N., Shirasawa, Y., Miyazaki, Y., Natori, M., Matunaga, S., Furuya, H. and Sakamoto, H., Mission report on the solar power sail deployment demonstration of IKAROS,", 52nd AIAA/ASME/ASCE/ AHS/ASC Structures, Structural Dynamics and Materials Conference, Denver, Colorado (2011), AIAA 2011-1887.

Senda, K., Ohta, S., Igarashi, Y., Watanabe, A., Hori, T., Ito, H., Tsunoda, H., and Watanabe, K., Deploy experiment of inflatable tube using work hardening, 47th AIAA/ASME/ASCE/AHS/ASC Structures, Structural Dynamics, and Materials Conf., Newport, RI (2006), AIAA 2006-1808.

Tanizawa, K. and Miura, K., Large displacement configurations of bi-axially compressed infinite plate, Trans. Japan Society for Aeronautical and Space Sciences, Vol.20, No.50 (1978), pp.177-187.

Watanabe, A., Ito, H. and Hori, T., Study of the extensible structure which coated a braid, Japanese 56th United Conf. Space Science and Technology (Ukaren), Beppu, Japan (2012), JSASS-2012-4496 (in Japanese). 